Fernando Ferrari Filho

\title{
Análise de um Motor do Ciclo Diesel operando no Modo Bicombustível: Diesel / Etanol
}

Dissertação apresentada como requisito parcial para obtenção do título de Mestre pelo Programa de PósGraduação em Engenharia Mecânica da PUC-Rio.

Orientador: Carlos Valois Maciel Braga

Co-orientador: Sergio Leal Braga

Rio de Janeiro 
Fernando Ferrari Filho

\section{Análise de um Motor do Ciclo Diesel operando no Modo Bicombustível: Diesel / Etanol}

Dissertação apresentada como requisito parcial para obtenção do título de Mestre pelo Programa de PósGraduação em Engenharia Mecânica da PUC-Rio. Aprovada pela Comissão Examinadora abaixo assinada.

Prof. Carlos Valois Maciel Braga

Orientador

Departamento de Engenharia Mecânica - PUC-Rio

Prof. Sergio Leal Braga Co-Orientador Departamento de Engenharia Mecânica - PUC-Rio

Prof. José Alberto dos Reis Parise Departamento de Engenharia Mecânica - PUC-Rio

Ph.D Julio César Cuisano Egúsquiza Departamento de Engenharia Mecânica - PUC-Rio

Prof. José Eugênio Leal

Coordenador Setorial do Centro Técnico Científico - PUC-Rio

Rio de Janeiro, 20 de dezembro de 2011 
Todos os direitos reservados. É proibida a reprodução total ou parcial do trabalho sem autorização da universidade, do autor e do orientador.

\section{Fernando Ferrari Filho}

Formado em Engenharia Mecânica pela Pontifícia Universidade Católica do Rio de Janeiro em dezembro de 2008. Atualmente trabalha na área de gerenciamento de projetos.

Ficha Catalográfica

Ferrari Filho, Fernando

Análise de um motor do ciclo diesel operando no modo bicombustível: diesel/etanol / Fernando Ferrari Filho; orientador: Carlos Valois Maciel Braga ; co-orientador: Sergio Leal Braga. - 2011.

118 f. : il. (color.) ; $30 \mathrm{~cm}$

Dissertação (mestrado) - Pontifícia Universidade Católica do Rio de Janeiro, Departamento de Engenharia Mecânica, 2011.

Inclui bibliografia.

1. Engenharia mecânica - Teses. 2. Motor diesel. 3. Etanol. 4. Liberação de calor. 5. Emissões. I. Braga, Carlos Valois Maciel. II. Braga, Sergio Leal. III. Pontifícia Universidade Católica do Rio de Janeiro. Departamento de Engenharia Mecânica. IV. Título.

CCD: 621 


\section{Agradecimentos}

À minha mãe, pelo incentivo, apoio e amor, que me deu forças para lutar e concretizar o presente trabalho.

Aos Professores Carlos Valois Maciel Braga e Sergio Leal Braga pelo grande incentivo e confiança depositada, mesmo com as grandes mudanças realizadas ao longo dos três últimos anos, acreditando em mim.

A PUC-Rio, pelos auxílios concedidos, sem os quais este trabalho não poderia ter sido realizado.

Ao doutor engenheiro mecânico Júlio Cesar Cuisano Egúsquiza pelo grande apoio e uma grande amizade.

A Promon Engenharia por incentivar e acreditar em mim, colaborando com os créditos educativos para a realização do mestrado.

Ao ITUC, Instituto Tecnológico da PUC-Rio, através dos funcionários administrativos, ressaltando Leandro Góis por sempre presente nas questões de informática. 


\section{Resumo}

Filho, Fernando Ferrari; Braga, Carlos Valois Maciel; Braga, Sergio Leal. Análise de um Motor do Ciclo Diesel Operando no Modo Bicombustível: Diesel / Etanol. Rio de Janeiro, 2011. 118p. Dissertação de Mestrado Departamento de Engenharia Mecânica, Pontifícia Universidade Católica do Rio de Janeiro.

Este trabalho avaliou o comportamento de um motor do ciclo Diesel, operando no modo original (Diesel puro) e no modo bicombustível (Diesel / etanol), em dois modos de hidratação do álcool (70 e 93 INPM). A rotação foi mantida fixa em 1800 rpm. A finalidade foi estudar os parâmetros de desempenho do motor e analisar a liberação de calor pela combustão, como também, o calor trocado com as paredes. Avaliou-se como parâmetros de desempenho, o rendimento térmico, consumo específico de combustível e emissão de poluentes. A fase inicial do trabalho constou de ensaios experimentais realizados no conjunto motor / dinamômetro nos modos mencionados acima. O objetivo foi coletar a variação de pressão no interior do cilindro, consumo de combustível, emissão de gases, temperaturas em pontos estratégicos, entre outros. Em uma segunda etapa foi realizada uma análise dos parâmetros de desempenho e da liberação de calor. Para emissões de poluentes, observou-se uma diminuição de MP em altas taxas de substituição. No entanto, notouse um aumento elevado de HC. Em baixas cargas e taxas de substituição elevadas houve redução de emissão de NOx. O rendimento térmico apresentou comportamentos similares em 70 e $93^{\circ}$ INPM. Em altas cargas e altas taxas de substituição houve um sensível aumento do rendimento quando comparado ao modo original. O rendimento foi menor para baixas cargas com altas taxas de substituição, em relação ao modo original. O início da combustão no modo bicombustível foi antecipado em relação ao modo original, nas condições de altas cargas e máximas taxas de substituição. Isto foi devido à liberação de calor que ocorreu mais cedo no modo bicombustível. Ressalta-se que, nas mesmas condições, houve a ocorrência de um maior calor trocado com as paredes do cilindro, em ambos os modos de hidratação (70 - 93 ${ }^{\circ}$ INPM), quando comparado ao modo original.

\section{Palavras-chave}

Motores Diesel; etanol; liberação de calor; emissões 


\section{Abstract}

Filho, Fernando Ferrari; Braga, Carlos Valois Maciel (Advisor); Braga, Sergio Leal (Co-Advisor). Evaluation of Diesel Cycle Engine Operating in the Dual Fuel Mode: Diesel / Ethanol. Rio de Janeiro, 2011. 118p. MSc. Dissertation Departamento de Engenharia Mecânica, Pontifícia Universidade Católica do Rio de Janeiro.

This work aimed to evaluate a Diesel cycle engine operating in the original (only Diesel) and dual-fuel modes (Diesel / ethanol) in two levels of hydration of alcohol (70 and $93^{\circ}$ INPM). Speed was kept fixed at $1800 \mathrm{rpm}$. The purpose was to study the parameters of engine performance and analyze the heat release by combustion and heat exchanged to the cylinder's walls. For parameters of performance, evaluation of thermal efficiency, specific fuel consumption and emissions were conducted. Initial activities consisted in trial tests on the engine / dynamometer in the two modes as mentioned above. The goal was to collect the variation of indicated cylinder pressure data, as well as fuel consumption, emissions and temperatures at strategic points. Secondly, performance parameters and heat release analysis was performed. For emissions, a decrease in PM was found at higher replacement rates; however, in the same condition a large increase in $\mathrm{HC}$ was obtained. At low loads and at higher replacement rates, NOx emissions were reduced. Thermal efficiency showed similar behavior at 70 and $93^{\circ}$ INPM. At high loads and at higher replacement rates a significant increase in thermal efficiency compared to the original mode and for low loads with higher replacement rates thermal efficiency was decreased. In high loads and at higher replacement rates conditions, the process of combustion occurred before in the dual fuel mode, due to earlier heat release compared to original mode (only Diesel). In the same conditions an increase of heat exchanged to the cylinder's wall in both modes of hydration of alcohol (70 and $93^{\circ}$ INPM) compared to the original mode was obtained.

\section{Keywords}

Diesel engines; ethanol; heat release; emissions. 


\section{Sumário}

1 Introdução 14

1.1. Evolução dos Motores Diesel 18

1.2. Motivação 19

1.3. Objetivo 21

1.4. Descrição da Dissertação 22

2 Revisão Bibliográfica e Tópicos Técnicos 23

2.1. Revisão Bibliográfica 23

2.2. Tópicos Técnicos 29

3 Método de Modelagem e Procedimento de Cálculo 42

3.1. Temperatura no Interior do Cilindro 46

3.2. Transferência de Calor pelas Paredes do Cilindro 47

3.3. Pressão sem Combustão 49

3.4. Procedimento de Cálculo 50

4 Procedimento Experimental e Redução de Dados 54

4.1. Motor Diesel 55

4.2. Pressão no Interior da Câmara 56

4.3. Softwares de Controle $\quad 57$

4.4. Injeção de Álcool 58

4.5. Smoke Meter 59

4.6. TESTO 59

$\begin{array}{ll}\text { 4.7. Potência } & 60\end{array}$

4.8. Rendimento Térmico 60

4.9. Consumo de Etanol 61

4.10. Consumo Específico de Combustível 61

4.11. Taxa de Substituição 62

4.12. Trabalho Indicado 63

4.13. Equações Geométricas 63 
5 Resultados e Discussões 66

5.1. Parâmetros de Desempenho 68

5.2. Trabalho Útil Gerado 76

5.3. Curva de Pressão no Interior do Cilindro 79

5.4. Temperatura Média no Interior do Cilindro 86

5.5. Calor Liberado no Processo de Combustão 91

5.6. Calor Perdido pelas Paredes do Cilindro 102

6 Conclusões e Recomendações $\quad 111$

$\begin{array}{ll}\text { Referências Bibliográficas } & 115\end{array}$ 


\section{Lista de figuras}

Figura 1: Oferta interna de energia (BEN, 2011) 17

Figura 2: Atraso de ignição, motor Diesel (Guibet, 1999) 35

Figura 3: Fases da combustão - motor Diesel (Heywood, 1988) 37

Figura 5: Bancada dinamométrica PUC-Rio 54

Figura 6: Motor Diesel MWM 55

Figura 7: Transdutor de pressão $\quad 57$

Figura 8: Star da AVL e software de controle PUMA 58

Figura 10: Equipamento de medição de poluentes 59

Figura 11: Conjunto cilindro-pistão (Heywood, 1988) 64

Figura 12: Pressão máxima por ciclo em 100\% de carga @ 93ㅇINPM 67

Figura 13: Pressão no interior do cilindro - ciclo 75, CoV(Pmáx) de 1\%

Figura 14: Rendimento térmico por taxa de substituição e carga do motor @ 1800 rpm com 70 e 93 INPM

Figura 15: Consumo específico de combustível por taxa de substituição e carga do motor @ 1800 rpm com 70 e $93^{\circ}$ INPM

Figura 19: Curva de pressão por volume em $100 \%$ de carga, 93INPM@ 1800 rpm 76

Figura 20: Pressão do cilindro em 100\% de carga - 93 INPM 79

Figura 21: Pressão do cilindro em 100\% de carga - 70 INPM 80

Figura 22: Pressão do cilindro em $75 \%$ de carga - 93 INPM 81

Figura 23: pressão do cilindro em $75 \%$ de carga - 70\% INPM 81

Figura 24: Pressão do cilindro em 50\% de carga - 93 INPM 82

Figura 25:Pressão do cilindro em 50\% de carga - 70 INPM 83

Figura 26: Pressão do cilindro em 25\% de carga - 93 INPM 84

Figura 27: Pressão do cilindro em 25\% de carga - $70^{\circ}$ INPM 84

Figura 29: Temperatura em 100\% de carga - 93 INPM 86

Figura 30: Temperatura em 100\% de carga - 70 INPM 87

Figura 31: Temperatura em 75\% de carga - $93^{\circ}$ INPM 87

Figura 34: Temperatura em $50 \%$ de carga $-70^{\circ}$ INPM 89

Figura 36: Temperatura em 25\% de carga $-70^{\circ}$ INPM 90 
Figura 37: Curva de liberação de calor em 100\% de carga -

$93^{\circ}$ INPM 92

Figura 38: Calor total liberado em 100\% de carga - $93^{\circ}$ INPM 92

Figura 40: Calor total liberado em 100\% de carga - 70 INPM 94

Figura 42: Calor total liberado em 75\% de carga - $93^{\circ}$ INPM 95

Figura 44: Calor total liberado em 75\% de carga - 70 INPM 96

Figura 46: Calor total liberado em 50\% de carga - 93 INPM 97

Figura 47: Curva de liberação de calor em $50 \%$ de carga -

$70^{\circ}$ INPM 98

Figura 48: Calor total liberado em 50\% de carga - $93^{\circ}$ INPM 99

Figura 49: Curva de liberação de calor em $25 \%$ de carga -

$93^{\circ}$ INPM 99

Figura 50: Calor total liberado em 25\% de carga - 93 INPM 100

Figura 51: Curva de liberação de calor em 25\% de carga -

$70^{\circ}$ INPM 101

Figura 52: Calor total liberado em 25\% de carga - 70 INPM 101

Figura 55: Calor instantâneo perdido em 100\% de carga - 70 INPM 103

Figura 57: Calor instantâneo perdido em 75\% de carga - 93 INPM 104

Figura 59: Calor instantâneo perdido em 75\% de carga - 70 INPM 105

Figura 61: Calor instantâneo perdido em 50\% de carga - 93 INPM 106

Figura 63: Calor instantâneo perdido em 50\% de carga - 70 INPM 107

Figura 65: Calor instantâneo perdido em 25\% de carga - 93 INPM 108

Figura 67: Calor instantâneo perdido em 25\% de carga - 70 INPM 109 


\section{Lista de tabelas}

Tabela 1: Consumo de Combustíveis - 2009 e 2010 (BEN 2011) 17

Tabela 2: Propriedades físico-químicas dos combustíveis (1 atm e $288 \mathrm{~K}$ ) 32

Tabela 3: Ficha técnica do motor 56

Tabela 4: Taxas de substituição em 70 e 93 INPM @ 1800 rpm 69

Tabela 5: Trabalho útil em 70 e 93 INPM @ 1800 rpm 77 


\section{Nomenclatura}

$\begin{array}{ll}(\mathrm{A} / \mathrm{C}) & \text { Razão ar-combustível } \\ (\mathrm{A} / \mathrm{C})_{\mathrm{s}} & \text { Razão ar-combustível estequiométrica } \\ \mathrm{B} & \text { Diâmetro do cilindro } \\ \mathrm{C} & \text { Carbono } \\ \mathrm{cec} & \text { Consumo específico de combustível (g/kW.h) } \\ \mathrm{CO} & \text { Monóxido de Carbono } \\ \mathrm{CO}_{2} & \text { Dióxido de Carbono } \\ \mathrm{CoV} & \text { Coeficiente de Variação } \\ \mathrm{C}_{\mathrm{p}} & \text { Calor específico a pressão constante } \\ \mathrm{C}_{\mathrm{v}} & \text { Calor específico a volume constante } \\ \mathrm{C}_{2} \mathrm{H}_{5} \mathrm{OH} & \text { Etanol } \\ \mathrm{C}_{12} \mathrm{H}_{26} & \text { Dodecano (Óleo Diesel leve) } \\ \mathrm{h} & \text { Coeficiente global de troca de calor }\left(\mathrm{W} / \mathrm{m}^{2} \mathrm{~K}\right) \\ \mathrm{H}_{2} \mathrm{O} & \text { Vapor d'água } \\ \mathrm{m}_{\mathrm{ar}} & \text { Massa de ar } \\ \mathrm{m}_{\mathrm{comb}} & \text { Massa de combustível } \\ \dot{m} & \text { Vazão mássica (kg/h) } \\ \mathrm{N} & \text { Velocidade angular (rpm) } \\ \mathrm{O}_{2} & \text { Oxigênio } \\ \mathrm{P} & \text { Potência } \\ \mathrm{PCl} & \text { Poder calorífico inferior (J/kg) } \\ \mathrm{PME} & \text { Pressão Média Efetiva (bar) } \\ \mathrm{R}_{\mathrm{ar}} & \text { Constante do ar (J/kg.K) } \\ \mathrm{T} & \text { Torque (N.m) } \\ \mathrm{TS} & \text { Taxa de substituição (\%) } \\ \mathrm{V}_{\mathrm{d}} & \text { Volume deslocado por ciclo (m³) } \\ \mathrm{W} & \text { Trabalho útil (J) } \\ & \end{array}$




\section{Subscritos e Letras Gregas}

$\begin{array}{ll}\text { e } & \text { Relativo à vazão mássica do etanol } \\ \rho & \text { Massa específica do } \operatorname{ar}\left(\mathrm{kg} / \mathrm{m}^{3}\right) \\ \eta_{t, d} & \text { Rendimento térmico no modo Diesel (\%) } \\ \eta_{t, D a} & \text { Rendimento térmico no modo Diesel-etanol (\%) } \\ \beta & \text { Emissividade } \\ \sigma & \text { Constante de Stefan-Boltzmann } \\ \Delta \theta & \text { Variação do ângulo do virabrequim } \\ \gamma & \text { Razão entre os calores específicos }\end{array}$

